\title{
Current Landscape (At March 2019) Of Chimeric Antigen Receptor T Cell Clinical Trials
}

\author{
Ortiz De Landazuri Iñaki ${ }^{1}$, Egri Natalia ${ }^{1}$, San Bartolomé Clara ${ }^{1}$, Español-Rego ${ }^{1}$, Marta ${ }^{1}$ and Juan Manel $^{1,2,3,4 *}$ \\ ${ }^{1}$ Servei d Immunologia
}

${ }^{2}$ Hospital Clínic de Barcelona IDIBAPS

${ }^{3}$ University of Barcelona

${ }^{4}$ Hospital Sant Joan de Déu Barcelona Spain

Submission: March 23, 2019; Published: May 21, 2019

*Corresponding author: Manel Juan, Servei d Immunologia -CDB Hospital Clínic de Barcelona c/ Villarroel 17008036 Barcelona Spain

Abstract

Chimeric Antigen Receptor (CAR) technology has shifted from the laboratory bench to the clinic due to promising adoptive antitumor results. The Food and Drug Administration (FDA) and European Medicines Agency (EMA) approval of tisagenlecleucel and axicabtagen ciloleucel was a milestone in the genetically engineered $\mathrm{T}$ cell therapies and encouraged the onset of clinical trials by the pharmaceutical industry and the National Institutes of Health (NIH) or universities worldwide. Hematologic malignancies are being targeted in the majority of interventional studies employing as targets several different surface antigens, such as anti-B cell maturation antigen (BCMA), CD19, CD20, CD22, CD23 and CD30 CAR T cells. On the other hand, cell surface antigens like GD-2, mesothelin, glypican-3 (GPC3) or c-MET are targets in many current solid tumor trials. In this study, we summarize the present landscape and some overall results in terms of CAR T cell-based clinical trials, with deep attention in the revision of the distinctions between the clinical trials sponsored by the pharmaceutical industry and those developed without this sponsorship.

Keywords: Chimeric Antigen Receptor; T cell; Immunotherapy

Abbrevations: CAR: Chimeric Antigen Receptor; FDA: Food and Drug Administration; EMA: European Medicines Agency; NIH: National Institutes of Health; BCMA: B Cell Maturation Antigen; GPC3: Mesothelin Glypican-3; TCR: T Cell Receptor; ALL: Acute Lymphoblastic Leukemia; DLCL: Diffuse Large B Cell Lymphoma; CLL: Chronic Lymphocytic Leukemia; MM: Multiple Myeloma; AML: Acute Myeloid Leukemia; NCI: National Cancer Institute

\section{Introduction}

Chimeric Antigen Receptor (CAR) T cells are emerging and highly promising adoptive antitumor immunotherapies based on patient $\mathrm{T}$ cells transduced with a synthetic receptor to recognize native surface antigens and redirect cytotoxicity of these $\mathrm{T}$ cells against the tumor. Although there is not a standard procedure to design CARs [1], their construct generally contains three separated modules. The extracellular domain is typically the scFv derived from a monoclonal antibody and shows the capability to recognize the desired tumor antigen, while the intracellular module contains activating signaling domains like $\mathrm{CD} 3 \xi$ and others. Between these regions, the transmembrane region hinges the CAR to the plasma membrane of the $\mathrm{T}$ cell. The better knowledge of the signaling pathways implicated in $\mathrm{T}$ cells proliferation, co stimulation and proliferation have made possible the development of different CAR T cell generations. The addition of a costimulatory domain like CD28 or CD137 (known as 4-1BB) to the first-generation CARs, with only $\mathrm{CD} 3 \xi$ as intracellular domain, lead to the second
CAR generation. This improvement increases T cells effectivity and persistence in patients. CAR third generation includes a third signaling domain in the intracellular module, but until now its advantage for antitumor efficacy is still unclear [2].

In contrast to other therapies employing engineered $\mathrm{T}$ cells like those with transgenic T Cell Receptor (TCR), the specificity of CART is not restricted to human leukocyte antigen, allowing a direct binding to cell-surface protein or even no-protein antigens. This advantage added to technical improvements in the development of methods to culture and transduce $\mathrm{T}$ cells accelerated the clinical interest in CART antitumor therapy [3], and attracted the attention of the pharmaceutical industry and other entities like National Institutes of Health (NIH) or universities worldwide in order to successfully introduce CAR T cell products. In fact, more and more interventional studies are being currently developed with CART immunotherapies. 
In this situation, tisagenlecleucel and axicabtagen ciloleucel (anti-CD19 CAR T cell treatments), developed by University of Pennsylvania and NIH researchers and supported by Novartis and Gilead, obtained Food and Drug Administration (FDA) and European Medicines Agency (EMA) approbation in 2017 and 2018 respectively, for the treatment of pediatric and young adult patients with CD19+ relapsed or refractory B cell acute lymphoblastic leukemia (ALL), and relapsed or refractory B cell non-Hodgkin lymphomas [diffuse large B cell lymphoma (DLCL) primary mediastinal B cell lymphoma, and transformed follicular lymphoma]. These drugs were studied in JULIET (NCT02445248) [4], ELIANA (NCT02435849) [5] and ZUMA-I (NCT02348216) [6] clinical trials targeting CD19, a molecule restricted and broadly expressed in B cells. Thus, CART19 treatments are currently considered therapeutic options for CD19+ hematologic malignancies with no responses to conventional treatments.

On the other hand, in the setting of CART therapy, the results in solid tumor clinical trials have not been as promising as expected yet $[7,8]$. However, novel knowledge in tumor microenvironment $[9,10]$ and the identification of new suitable target antigens [11] are becoming the basis in the presentday solid tumor clinical trials. The aim of this mini-review is to describe the actual landscape and global results in terms of CAR $\mathrm{T}$ cell-based clinical trials worldwide, with special interest in the revision of the differences between the clinical trials sponsored by the pharmaceutical industry and those developed without this sponsorship.

\section{Materials and Methods}

The evaluation was performed with data obtained from clinicaltrials.gov database analyzing privately, and publicly funded clinical studies conducted around the world. Several keywords were used to obtain the information and evaluation of items was defined by comparing the presenting information. Only interventional studies with CART treatment that were in recruiting, not yet recruiting, active but not yet recruiting or already completed status, were took in consideration for the revision and sorted depending on funder type and by topic. CAR T cell therapies for hematologic malignancies and for solid tumors were separately evaluated.

\section{Results}

From our evaluation we have been able to analyze the worldwide distribution of CAR T cell clinical trials, defining also specific features of the type of cancer targeted. Trials with CAR $\mathrm{T}$ cells therapy for hematologic malignancies were separately examined from solid tumor interventional studies and most frequently cell surface antigens were commented for each one.

\section{Worldwide distribution of CAR T cell clinical trials}

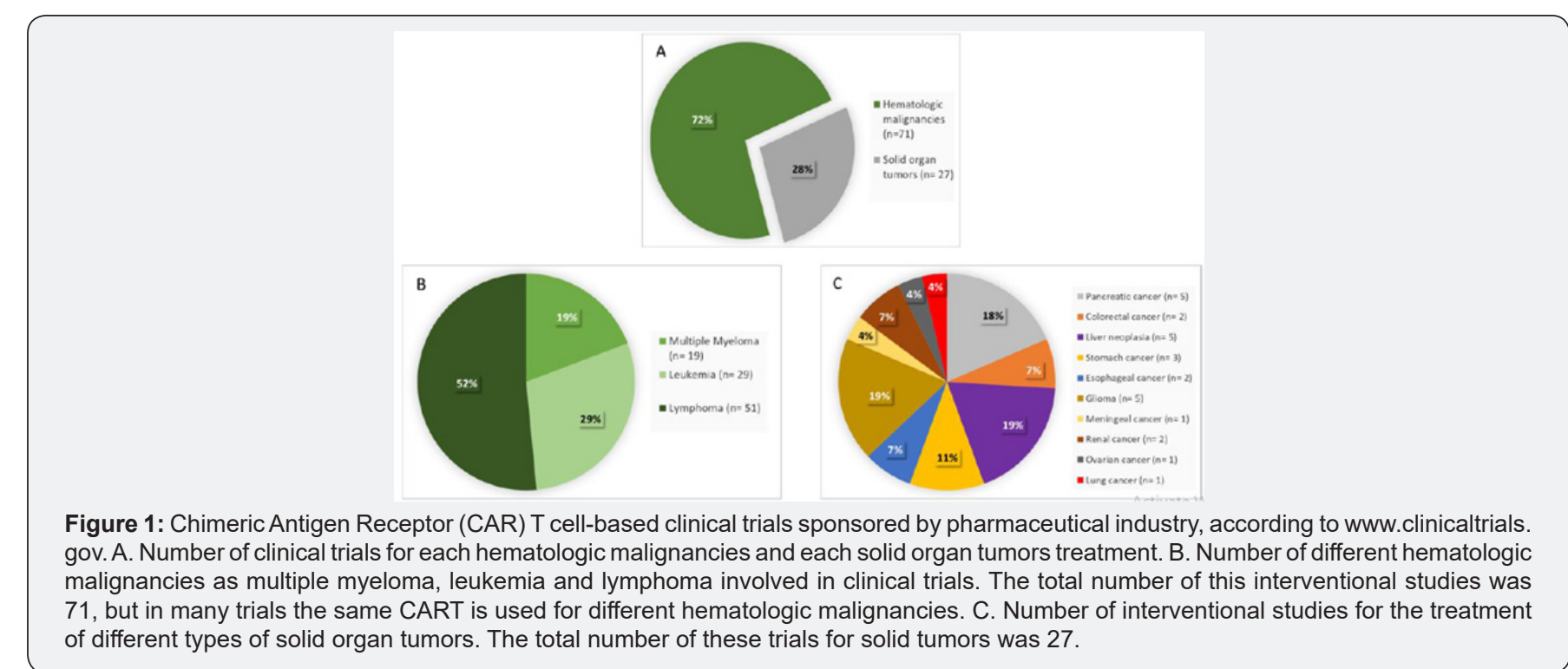

The technical issue to design CAR constructions and the exciting results obtained in trials of patients with CD19+ B malignancies [4-6, 12,13] have greatly impacted in the clinical trial scenario. Nowadays, more than 200 active clinical trials are registered in www.clinicaltrials.gov, using CAR T cell treatment for any tumor. From them, almost 100 are being sponsored by the pharmaceutical industry (Figure 1) while nearly 130 are being developed by the NIH, other U.S. Federal agency, universities or organizations (Figure 2). The global distribution of this clinical trial is really unequal, being the U.S. and China the countries where the number of clinical trials is higher, reaching in both states more than 100 interventional studies. The EU follows them, but by far, with nearly 20 clinical trials. The pharmaceutical industry also concentrates their CART based clinical trials in the U.S. and China, with a higher number of Chinese trials. On the other hand, the trend has reversed in not industry-sponsored interventional studies, with more trials in the U.S. in comparison to China. Although solid cancer tumors represent the majority of human 
cancer, B cell malignancies are the most common diseases tested in this sponsored or not by industry trials. In any case there are

an increasing number of CART clinical trials implementing similar strategies for solid tumors with some promising outcomes.

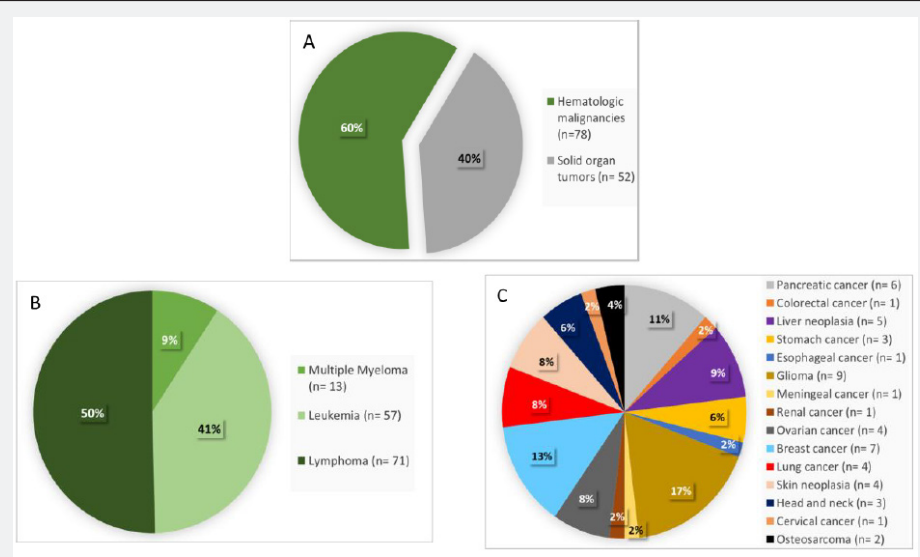

Figure 2: Chimeric antigen receptor (CAR) T cell-based clinical trials not sponsored by pharmaceutical industry, according to www. clinicaltrials.gov. A. Number of clinical trials for hematologic malignancies and solid organ tumors treatment. B. Number of different hematologic malignancies as multiple myeloma, leukemia and lymphoma involved in clinical trials. The total number of this interventional studies was 78, but in many trials the same CART is used for different hematologic malignancies. C. Number of interventional studies for the treatment of different types of solid organ tumors. The total number of these trials for solid tumors was 52 .

\section{CAR T cell therapy for hematologic malignancies}

The number of interventional studies employing CART for B cell proliferative diseases is rapidly growing due to the excellent capability of $\mathrm{T}$ cells to reach the lymph nodes, bone marrow and blood [14]. Moreover, the selection of the target molecule is not tough as this hematologic malignancy are routinely sorted according to cell surface antigens.

The FDA and EMA approval of tisagenlecleucel [4,5] and axicabtagen ciloleucel [6] was a milestone in the genetically engineered $\mathrm{T}$ cell therapies, encouraging the onset of clinical trials based on described B cell surface antigens like CD19, CD20, CD22, CD23, CD30 or CD269 [better known as B cell maturation antigen (BCMA)] or other antigens that have been targeted in preclinical studies [15]. CD20 is an over-expressed lectin on ALL and on mature lymphoid neoplasia, that has already been targeted to treat advanced DLCL [16]. CD22 is mainly detected on tumor B cells and is less expressed than CD20 on normal B cells [17]. CD23 is a B cell activation marker that can be a good target for the treatment of chronic lymphocytic leukemia (CLL) [18]. CD30 is globally expressed in classical Hodgkin lymphomas, anaplastic large cell lymphomas, and in some other lymphomas like cutaneous T cell lymphomas or DLCL [19]. CD269 (BCMA) is expressed on late-B cells and plasma cells and is highly expressed in patients with Multiple Myeloma (MM) [20].

Treatments for hematologic malignancies are the objective in more than half amount of total CART clinical trials. This aim is clear in industry-sponsored trials, representing a $72 \%$ of these funder type studies (Figure 1A). In non-industry-sponsored interventional studies this value reaches the $60 \%$ (Figure 2A). Most of them are enrolling patients that do not respond to conventional therapies or with relapsed disease after a hematopoietic transplant with a poor prognostic. Lymphoma is the most common studied hematologic malignancy in these trials, independently of the funder type (Figure 1B, 2B). Leukemia and MM are also remarkable diseases in terms of number of interventional studies. In China, two clinical trials employing CART19 (NCT02976857, NCT03811457) have been completed with the sponsorship of Cellular Biomedicine Group Ltd. and UWELL Biopharma, respectively. The first one is a phase 1 study, planned to evaluate the safety and efficacy in the treatment of refractory DLCL in adults and older adults and the other included patients with relapsed or refractory CD19+ leukemia and lymphoma up to 70 years. However, their results are not yet posted. Although variations of CART19 are still focusing the aims of many trials, novel different CART treatments are being also developed. In fact, Dana-Farber Cancer Institute sponsored by Celyad (Cardio3 BioSciences) has completed a phase 1 clinical trial (NCT02203825) which evaluates CAR T cells targeting NKG2D-ligands on the surface of cancer cells. This study was designed for patients with Acute Myeloid Leukemia (AML) and with relapsed or refractory MM with progressive disease and provides interesting information for further optimization of NKG2D CAR T cell manufacturing [21]. Moreover, the strategy of using anti-BCMA CAR T cells is now being studied in almost 20 multicenter clinical trials sponsored by industry with the collaboration of, i.e., Mayo Clinic Cancer Center (NCT03548207), Memorial Sloan Kettering Cancer Center (NCT03070327), University of California (KarMMa, NCT03361748) or Peking University Third Hospital (NCT03758417). These phase 1 and 2 clinical trials are recruiting people older than 18 years with a diagnosis of MM, with an estimated enrollment that ranges from 36 to 150 participants. Participants will receive BCMA-targeted CAR T cells as a single infusion after lymphodepletion; but dose level are different depending on the trial. In KarMMA, i.e., they will be infused at a dose ranging from 15-45x107 CAR+ T cells after receiving the lymphodepleting chemotherapy. 
Anti-CD30 CAR $\mathrm{T}$ cells for treating CD30-expressing hematologic malignancies are being tested in some nonindustry-promoted clinical trials that are recruiting patients (NCT02917083, NCT01316146) or are active but not recruiting yet (NCT01316146) for the treatment of relapsed or refractory Hodking's or non-Hodgking's lymphoma. The RELY-30 interventional study (NCT02917083) of Baylor College of Medicine and Houston Methodist Hospital is a phase 1 study with an estimated enrollment of 12 patients, including those with an age between 12 and 75. Although the overall response rate is estimated in a time frame of 8 weeks, the duration of this study is estimated to be extended to 15 years because median and mean number of $\mathrm{T}$ cells transduced with the anti-CD30 vector in that time wanted to be observed. In the same center, and also without an industry sponsorship, the CHARKALL clinical trial (NCT00881920) is going to evaluate, in nearly 50 patients, CAR T cell treatment targeting the kappa light chain of immunoglobulin expressed in patients with CLL, B cell lymphoma or MM.

\section{CAR T cell therapy for solid tumors}

CAR $\mathrm{T}$ cell-based therapy for solid tumor treatment was originally tested in clinical trials at the beginning of the last decade, but the initial results were not really promising [22-24]. Moreover, a mortal adverse event of pulmonary toxicity employing HER2-targeted CAR T cells was reported in a interventional study for patients with colon cancer [25]. This toxicity was ascribed to HER2 expression in the lung epithelium. In contrast to hematologic malignancies, solid tumors show certain characteristics that impair the CAR T cells efficacy. The efficacy of this T cell treatment is challenged by the immunosuppressive microenvironment and the difficulty to reach the tumor, survive and proliferate. However, the main limitations is the election of a specific cell surface antigen to target with the CAR because solid tumors are usually classified depending on anatomic or histological properties instead of with the surface antigen expression profile. This limitation is trying to be overcome with multiple preclinical studies in order to find antigens that are specifically expressed in different solid tumor types, but not in normal cells [11]. Many targets are being evaluated for solid malignancies like mesothelin, over-expressed in mesothelioma and lung, pancreas, breast, ovarian, and other cancers [26]. The ganglioside GD-2 is also being targeted as its consistently expression in neuroblastoma tumors [27]. Glypican-3 (GPC3) is an oncofetal protein which is highly expressed on some pediatric embryonal tumors like the majority of hepatoblastomas [28]. c-MET is a tyrosine kinase receptor for the hepatocyte growth factor (HGF) that is aberrantly expressed in various malignancies like hepatocellular carcinoma or gastrointestinal cancer [29].

There are now nearly 80 interventional studies testing CAR T cells for the treatment of different solid tumors. Gliomas, pancreatic and liver cancer are the most represented tumors in these trials (Figure 3 ). While only the $28 \%$ of industry-sponsored clinical trials are testing CAR T cells for solid tumors, the percentage increase up to the $40 \%$ in those trials that are not financed by industry (Figure 1C, 2C). The National Cancer Institute (NCI) and the Fuda Cancer Hospital have completed without industry sponsorship two trials of anti-GD2 CAR T cells in patients with GD2+ tumors. The NCI clinical trial (NCT02107963) is a phase 1 trial for children and young adults (1 to 35 years) with osteosarcoma, neuroblastoma or melanoma judged to be incurable with standard therapy because they are unresectable, metastatic, progressive or persistent or recurrent. The aim to assess the safety of administering escalating doses of anti-GD2 CAR T cells in those patients. The purpose of Fuda Cancer Hospital study (NCT03252171) was to evaluate the safety and effectiveness of this therapy in GD2 positive glioma patients However, their results are not yet posted. Results are also not posted in a completed trial of the Abramson Cancer Center, in University of Pennsylvania (NCT02159716), in which the target of the CAR T cell was the mesothelin in order to treat patients with metastatic pancreatic (ductal) adenocarcinoma, epithelial ovarian cancer and malignant epithelial pleural mesothelioma.

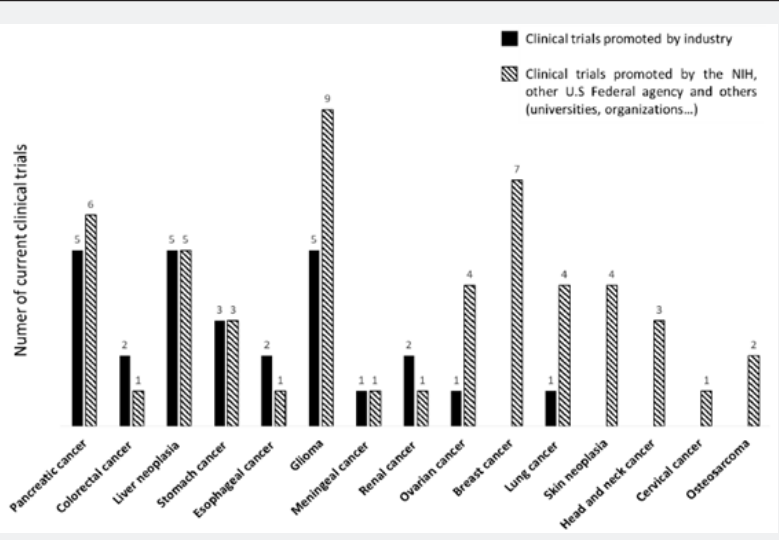

Figure 3: Chimeric antigen receptor (CAR) T cell-based clinical trials for solid tumor treatment. The plain colored bars represent clinical trials promoted by industry and the striped colored bars indicate clinical trials promoted by the National Health Institutes (NIH), other U.S. Federal agencies and other entities like universities and organizations. The total number of these trials for solid tumors was 79 . Data available in www.clinicaltrials.gov.

There are, at least 5 types of solid tumors that are not being evaluating for CAR T cell therapy in industry-sponsored clinical trials in comparison to non-industry-sponsored studies (Figure 3). This includes breast cancer, skin neoplasia, head 
and neck cancer, osteosarcoma and cervical cancer. In the case of breast cancer, there are 7 studies worldwide. The Memorial Sloan Kettering Cancer Center, i.e. in collaboration with the U.S. Department of Defense is recruiting patients in a phase 1 clinical trial (NCT02792114) that want to find a safe dose of mesothelintargeted T cells to treat metastatic HER2-negative breast cancer. The University of Pennsylvania is also testing in a pilot study to evaluate the feasibility, safety, and preliminary evidence of efficacy for intravenously administered, RNA electroporated autologous T cells expressing c-MET CAR in patients with advanced melanoma or breast carcinoma.

On the other hand, the pharmaceutical industry is making their bigger efforts in glioma, liver and pancreatic tumors. A study (NCT03146234) designed to determine the safety and efficacy of CAR-GPC3 $\mathrm{T}$ cells in patients with relapsed or refractory hepatocellular carcinoma is recruiting over 20 patients in RenJi Hospital with the sponsorship of Carsgen Therapeutics, Ltd. In that case, classical $3+3$ dose escalation will be applied in 20 participants to subsequent subjects based on the self-controlled dose escalation study.

\section{Discussion and Conclusion}

In the past few decades, CAR technology has shifted from the laboratory bench to the clinic due to the feasibility and efficacy. In this study, we summarized the current clinical studies on CART immunotherapy landscape and some characteristics of clinical trials sponsored by the pharmaceutical industry and those developed without this sponsorship. China and U.S. almost completely fill the scenario of this interventional studies, with few differences in the type of tumor treated taking into account the financing of each trial. Hematologic malignancies like MM, ALL or DLCL are being targeted in the majority of clinical trials employing anti-BCMA, CD19, CD20, CD22, CD23 and CD30 CAR T cells, independently of the funder type but with a higher number in the industry sponsored studies. CART19 treatment for lymphoma and leukemia is the most tested therapy in these studies, without considering clinical trial funder type. Targeting BCMA in MM is also an emerging strategy [20]. On the other hand, the pharmaceutical industry sponsors less number of clinical trials targeting cell surface antigens of solid tumors in comparison to the NIH, other U.S. Federal agencies, universities or organizations. Without considering the sponsorship, pancreatic or liver cancer and glioma are being the most targeted solid tumors in these trials. However, the number of studies with breast or ovarian cancer, or skin neoplasia as targeted diseases is higher in clinical trials promoted by the NIH, other U.S. Federal agencies or universities. The same applies when the immunotherapy is for treating lung or cervical cancer, osteosarcoma or head and neck cancer. Among others, the targeted cell surface antigens vary from GD-2, mesothelin or GPC3 to c-MET [26-29].

Although these interventional studies are demonstrating that CAR T cell treatment for B cell malignancies is feasible, the optimal design and manufacturing procedure or the CAR transfection vector remains to be defined. Every institution has developed a different manufacturing strategy and the patient admission criteria heterogeneously vary, as well as the target tumor surface antigen selected change depending on the antigen profile signature of the treated tumor. Thus, an objective comparison between CAR $\mathrm{T}$ cell therapies used in different trials is really tough in terms of efficacy and safety. Moreover, every CAR T cell therapy needs to be personalized performed from autologous leukapheresis. Thus, universal allogeneic $\mathrm{T}$ cells are needed for the preparation of universal CART (UCART) that can use as readyto-use therapeutic treatments for large-scale clinical applications [30]. Moreover, this therapy employs cytotoxic procedures to deplete lymphocytes before the CAR T cell infusion and, in some cases, this therapy leads to a dramatic response known as Cytokine Release Syndrome (CRS) characterized by fever, hypotension, cardiac and hepatic failure or, even, death [31-34].

Preclinical improvements in the development of CAR technology are allowing the achievement of better clinical results in many solid tumor trials, but there are still many handicaps to overcome. Data obtained with xenografted human tumors in mouse models suggest sustain that homing to tumor is possible [35], but this mice models do not exactly replicate neither every single human solid tumor feature nor the immune microenvironment in humans. The interaction between $\mathrm{T}$ cells and tumor cells in the suppressive tumor microenvironment and the required improvement in the CAR T cell homing, as well as the toxicity avoidance need further research and efforts.

\section{Acknowledgement}

This mini review is included as a work sponsored by grants PI18/000775 from Instituto de Salud Carlos III.

\section{References}

1. Sadelain M, Brentjens R, Rivière I (2013) The Basic Principles of Chimeric Antigen Receptor Design. Cancer Discov 3(4): 388-398.

2. Savoldo B, Ramos CA, Liu E, Mims MP, Keating MJ, et al. (2011) CD28 costimulation improves expansion and persistence of chimeric antigen receptor-modified T cells in lymphoma patients. J Clin Invest 121(5): 1822-1826.

3. June CH, Blazar BR, Riley JL (2009) Engineering lymphocyte subsets: Tools, trials and tribulations. Nat Rev Immunol 9(10): 704-716.

4. McGuirk JP, Fleury I, Borchmann P, Jaglowski S, Bachanova V, et al. (2018) Tisagenlecleucel in Adult Relapsed or Refractory Diffuse Large B-Cell Lymphoma. N Engl J Med 380(1): 45-56.

5. Verneris MR, June CH, Myers GD, Krueger J, Boyer M, et al. (2018) Tisagenlecleucel in Children and Young Adults with B-Cell Lymphoblastic Leukemia. N Engl J Med 378(5): 439-448.

6. Locke FL, Ghobadi A, Jacobson CA, Miklos DB, Lekakis LJ, et al. (2019) Long-term safety and activity of axicabtagene ciloleucel in refractory large B-cell lymphoma (ZUMA-1): a single-arm, multicentre, phase 1-2 trial. Lancet Oncol 20(1): 31-42.

7. Yu S, Li A, Liu Q, Li T, Yuan X, Han X, et al. (2017) Chimeric antigen receptor T cells: a novel therapy for solid tumors. J Hematol Oncol 10(1): 78.

8. Song X, Cao C, Li F, Yu H, Jin Y, Jiang Q, et al. (2017) CARTs for Solid Tumors: Feasible or Infeasible? Oncol Res Treat 40(9): 540-546. 
9. Wu T, Dai Y (2017) Tumor microenvironment and therapeutic response. Cancer Lett 387: 61-68.

10. Hirata E, Sahai E (2017) Tumor Microenvironment and Differential Responses to Therapy. Cold Spring Harb Perspect Med 7(7): a026781.

11. Cheever MA, Allison JP, Ferris AS, Finn OJ, Hastings BM, et al. (2009) The prioritization of cancer antigens: A National Cancer Institute pilot project for the acceleration of translational research. Clin Cancer Res 15(17): 5323-5337.

12. Kalos M, June CH, Levine BL, Bagg A, Porter DL (2011) Chimeric Antigen Receptor-Modified T Cells in Chronic Lymphoid Leukemia. N Engl J Med 365(8): 725-733.

13. Heller G, Yeh R, Przybylowski M, Frattini M, Usachenko Y, et al. (2011) Safety and persistence of adoptively transferred autologous CD19targeted $\mathrm{T}$ cells in patients with relapsed or chemotherapy refractory B-cell leukemias. Blood 118(18): 4817-4828.

14. Mastaglio S, Casucci M, Oliveira G, Cieri N, Bonini C, et al. (2013) Adoptive immunotherapy with genetically modified lymphocytes in allogeneic stem cell transplantation. Immunol Rev 257(1): 165-180.

15. Gill S, Maus MV, Porter DL (2016) Chimeric antigen receptor T cell therapy: 25 years in the making. Blood Rev 30(3): 157-167.

16. Zhang W, Wang Y, Han Q, Bo J, Guo Y, et al. (2014) Effective response and delayed toxicities of refractory advanced diffuse large B-cell lymphoma treated by CD20-directed chimeric antigen receptor-modified T cells. Clin Immunol 155(2): 160-175.

17. Till BG, Raubitschek AA, Wang J, James SE, Greenberg PD, et al. (2014) Antigen Sensitivity of CD22-Specific Chimeric TCR Is Modulated by Target Epitope Distance from the Cell Membrane. J Immunol 180(10): 7028-7038.

18. Marin V, Bertilaccio MTS, Tettamanti S, Pizzitola I, Parma M, Giordano Attianese GMP, et al. In vitro and in vivo model of a novel immunotherapy approach for chronic lymphocytic leukemia by antiCD23 chimeric antigen receptor. Blood 117(18): 4736-4745.

19. Grover NS, Savoldo B (2019) Challenges of driving CD30-directed CAR-T cells to the clinic. BMC Cancer 19(1): 203.

20. Cho SF, Anderson KC, Tai YT (2018) BCMA CAR T-cell therapy arrives for multiple myeloma: a reality. Ann Transl Med (2018) 6(S2): S93-S93.

21. Reder J, Murad JM, Daley H, Lehmann F, Dranoff G, et al. (2018) Manufacturing development and clinical production of NKG2D chimeric antigen receptor-expressing $\mathrm{T}$ cells for autologous adoptive cell therapy. Cytotherapy 20(7): 952-963.

22. Dudley ME, Wunderlich JR, Yang JC, Sherry RM, Topalian SL, et al. (2005) Adoptive cell transfer therapy following non-myeloablative but lymphodepleting chemotherapy for the treatment of patients with refractory metastatic melanoma. J Clin Oncol 23(10): 2346-2357.
23. Kershaw MH, Westwood JA, Parker LL, Wang G, Eshhar Z, et al. (2006) A Phase I Study on Adoptive Immunotherapy Using Gene-Modified T Cells for Ovarian Cancer. Clin Cancer Res 12(20): 6106-6115.

24. Lamers CHJ, Sleijfer S, Vulto AG, Kruit WHJ, Kliffen M, et al. (2006) Treatment of Metastatic Renal Cell Carcinoma with Autologous T-Lymphocytes Genetically Retargeted Against Carbonic Anhydrase IX: First Clinical Experience. J Clin Oncol 24(13): e20-2.

25. Morgan RA, Yang JC, Kitano M, Dudley ME, Laurencot CM, et al. (2010) Case Report of a Serious Adverse Event Following the Administration of T Cells Transduced with a Chimeric Antigen Receptor Recognizing ERBB2. Mol Ther 18(4): 843-851.

26. Morello A, Sadelain M, Adusumilli PS (2016) Mesothelin-targeted CARs: Driving T cells to solid Tumors. Cancer Discov 6(2): 133-146.

27. Straathof K, Thomas S, Anderson J, Pule M, Himoudi N (2016) An Optimized GD2-Targeting Retroviral Cassette for More Potent and Safer Cellular Therapy of Neuroblastoma and Other Cancers. PLoS One 11(3): e0152196.

28. Ortiz MV, Roberts SS, Glade Bender J, Shukla N, Wexler LH, et al. (2019) Immunotherapeutic Targeting of GPC3 in Pediatric Solid Embryonal Tumors. Front Oncol 9:289-291.

29. Mo HN, Liu P (2017) Targeting MET in cancer therapy. Chronic Dis Transl Med 3(3): 148-153.

30. Zhao J, Lin Q, Song Y, Liu D (2018) Universal CARs, universal T cells, and universal CAR T cells. J Hematol Oncol 11(1): 132.

31. Frey N (2017) Cytokine release syndrome: Who is at risk and how to treat. Best Pract Res Clin Haematol 30(4): 336-340.

32. Brudno JN, Kochenderfer JN (2016) Toxicities of chimeric antigen receptor T cells: recognition and management. Blood 127(26): 33213330.

33. Fitzgerald JC, Weiss SL, Maude SL, Barrett DM, Lacey SF, et al. (2017) Cytokine Release Syndrome After Chimeric Antigen Receptor T Cell Therapy for Acute Lymphoblastic Leukemia. Crit Care Med 45(2): e124-131.

34. Gödel P, Shimabukuro-Vornhagen A, von Bergwelt-Baildon M (2018) Understanding cytokine release syndrome. Intensive Care Med 44(3): 371-373.

35. Moon EK, Carpenito C, Sun J, Wang LCS, Kapoor V, et al. (2011) Expression of a functional CCR2 receptor enhances tumor localization and tumor eradication by retargeted human $\mathrm{T}$ cells expressing a mesothelin-specific chimeric antibody receptor. Clin Cancer Res 17(14): 4719-4730. 
Your next submission with Juniper Publishers will reach you the below assets

- Quality Editorial service

- Swift Peer Review

- Reprints availability

- E-prints Service

- Manuscript Podcast for convenient understanding

- Global attainment for your research

- Manuscript accessibility in different formats ( Pdf, E-pub, Full Text, Audio)

- Unceasing customer service

Track the below URL for one-step submission https://juniperpublishers.com/online-submission.php 